\title{
Aeroelastic Vibrations and Stability in Cyclic Symmetric Domains
}

\author{
BERNARD LALANNE $^{\mathrm{a}, *}$ and MAURICE TOURATIER ${ }^{\mathrm{b}}$ \\ a Turboméca, 64511 Bordes, France; ${ }^{\mathrm{b}} L M^{2} S$, UPRES A 8007, ENSAM, \\ 151 Bd de l'Hôpital, 75013 Paris, France
}

(Received 13 June 1997; Revised 10 March 1999; In final form 12 June 1999)

\begin{abstract}
This paper deals with rotating cyclic symmetric structures, immersed in light fluid flow. Firstly, general and usual cyclic symmetric properties are recovered from the Floquet theorem for differential equations, having space periodic coefficients in conjunction with a discrete space Fourier series development. The approach for aeroelastic problem having cyclic symmetry is then formulated based on the twin mode approach. In addition to modal one, rotating and stationary wave bases are introduced to derive the equilibrium equations for a non-dissipative system subjected to aerodynamic loading. Rotating wave basis is the natural one, and it also permits consistently to prescribe the aerodynamic pressure on the boundary between the fluid and the structure. The aerodynamic load is then derived from a harmonic analysis of the fluid flow extending to turbomachinery as in the case of aeroplane wing. In this way, aerodynamic forces may be obtained as general as required, depending on successive time derivatives of degrees of freedom in addition to themselves. Finally, some special cases are given and stability is studied for a cyclic periodic blade assembly, even when mistuning between sectors can occur.
\end{abstract}

Keywords: Cyclic symmetry, Forced vibrations, Aeroelasticity, Stability, Mistuning

\section{INTRODUCTION}

Vibration mastery is an important issue for compressors and turbines of turbomachines. These structures in general have circumferential periodic symmetry. It is well known that the studies of vibration mode of cyclic symmetric structures can be reduced by analysing one repeated sector, see Balasubramanian et al. (1993), Lalanne and
Touratier (1996), Mézière (1994), Thomas (1974), Wagner and Griffin (1994). In a turbomachinery, turbines are, among others, loaded by the fluid flow and the corresponding vibration problem of turbine may be solved using cyclic symmetric properties if the fluid flow satisfies these ones.

Hereafter, an analysis of fluid-structure vibration analysis for cyclic symmetric structures is presented. The cyclic symmetric decomposition for

* Corresponding author. Tel.: 0559125000 . Fax: 0559531512. 
linear problems is recovered by using the Floquet theorem. The principle of virtual work for the whole structure deals with the partial differential equations, having space periodic coefficients. Then, using Fourier series development of all the fields, the sector formulation for cyclic symmetric problems can be easily deduced from either continuous or discrete approaches, see Lalanne and Touratier (1996). To analyse aeroelastic responses, many ways are possible, see for example Wagner and Griffin (1994). Here, stationary and rotating wave bases are introduced in conjunction with the twin mode approach, Ewins (1988), by taking into account aeroelastic forces from a harmonic analysis of the fluid flow. Generalized forces associated to aerodynamic loading are then expanded as functions of generalized co-ordinates and their successive time derivatives. Examples are given wherein generalized co-ordinates and their first time derivatives are kept, and some particular cases are studied based on a weak aeroelastic coupling. Finally, stability analysis is presented for an aeroelastic problem associated with a blade assembly wherein a small mistuning (see for example Wei and Pierre, 1988) between blades has occurred. The effect of mistuning on stability is analysed based on a perturbation technique.

\section{BASIC FORMULATION OF CYCLIC SYMMETRIC PROBLEMS}

Let $\Omega$ be a circumferentially periodic structure around the $z$-axis in cylindrical co-ordinates $(r, \theta, z)$ and $\Omega_{\mathrm{s}}$, one repeated sector allowing to generate $\Omega$ by $\alpha$-rotation around the $z$-axis. Typically, $\Omega$ can be decomposed into $N$ sectors $\Omega_{\mathrm{s}}$. Let us consider small linearly varying displacements for the structure $\Omega$. The latter being circumferentially periodic, by applying the principle of virtual work it is clear that linear second-order partial differential equations are derived, with periodic coefficients. Thus, the Floquet theory is available, see Ince (1956), Stocker (1992) to take into account of the cyclic periodicity. Let $u(r, \theta, z, t)$ be then the three-dimensional displacement field in cylindrical co-ordinates, and $t$ the time. Recalling the closed condition associated to the circumferential periodicity as $u(r, \theta+2 \pi, z, t)=u(r, \theta, z, t)$, then the Floquet theory allows to write (Touratier, 1986)

$$
\begin{aligned}
u(r, \theta+\alpha, z, t) & =\exp (j q \alpha) u(r, \theta, z, t), \\
j^{2} & =-1 ; q=0,1, \ldots, N-1,
\end{aligned}
$$

where $q$ is the Floquet wave number.

Since deduced motion equations are linear and second order, with periodic coefficients, it is convenient to search the $u$-unknown from a Fourier series development with respect to the circumferential $\theta$-variable (Balasubramanian et al., 1993):

$$
\begin{aligned}
u(r, \theta, z, t) & =\sum_{k=-\infty}^{\infty} u_{k}(r, z, t) \exp (j k \theta) \\
& =\sum_{n=0}^{\tilde{N}} \sum_{k=-\infty}^{\infty} u_{k N \pm n}(r, z, t) \exp j(k N \pm n) \theta
\end{aligned}
$$

This equation may be reduced to

$$
\begin{gathered}
u(r, \theta, z, t)=\sum_{n=0}^{+\tilde{N}}\left(u_{n}(r, \theta, z, t)+u_{-n}(r, \theta, z, t)\right), \\
\tilde{N}=\frac{N}{2} \text { if } N \text { even, } \tilde{N}=\frac{N-1}{2} \text { if } N \text { odd }
\end{gathered}
$$

Introducing the $s$-index attached to any sector $\Omega_{\mathrm{s}}$, from Eqs. (3) and (1) we may still write

$$
u_{ \pm n}(r, \theta+s \alpha, z, t)=\exp ( \pm j n s \alpha) u_{ \pm n}(r, \theta, z, t)
$$

Replacing Eqs. (3) and (4) into the principle of virtual work, expected equations of motion are then deduced. Finally, we could demonstrate that the solution of the given boundary value problem for circumferentially periodic structures is reduced to solve $N$ uncoupled problems $P_{(n)}$ (identically $P_{(-n)}$ associated to the phase difference index- $n$ ) on the reference sector $\Omega_{\mathrm{o}}$, for each Fourier series component $u_{n}$ (identically $u_{-n}$ ) of the displacement $u$ 
which satisfies cyclic periodic properties. These conclusions are also achieved when using a discrete approach (finite elements for example) to solve the boundary value problem.

\section{THE AEROELASTIC PROBLEM}

Our interest is now for a rotating circumferentially symmetric structure in a fluid flow. Firstly, we consider a cyclic symmetric structure excited by a fluid flow which does not respect cyclic symmetric conditions. Then, excitation by the fluid flow is induced by the vibration of the structure, and this is called the aeroelastic effect. From the above paragraph, it is clear that results for the boundary value problem are still available if cyclic symmetric conditions are satisfied, as consistently assumed by Lane (1956). To be complete, it is necessary to include rotatory effects as in Childs (1993) and to give the aerodynamic loading, to be included into the boundary conditions (pressure).

However, gyroscopic effects will be hereafter neglected. From those latter, coupling between fluid and structure will be prescribed. Aerodynamic loading (pressure on external surfaces of turbine) may be characterized from experimental, analytical or purely numerical ways. Wind tunnel is needed for experimentally achieving the bench tests on rotor or cascade to deduce pressure on it, while analytical computations using a cascade model (JacquetRichardet and Henry, 1994) give approximate expressions for the total (steady and unsteady) pressure acting on blades of a turbine. The most complete and accurate approach deals with the numerical computations of the fluid flow (Marshall and Imregun, 1996) within steady and unsteady state phases. Anyway, the aerodynamic solution of the problem is given by reference to rotating wave basis.

Here, to model aeroelastic forces, a harmonic analysis of the fluid flow is made, prescribing at the fluid-structure, the boundary motion as in Poiron (1995) for aeroplane wing. The following expressions for displacements in rotating wave basis are considered assuming harmonic motions:

$$
\begin{aligned}
u_{n}^{l} & =\left(\hat{u}_{n}^{\prime l}+j \hat{u}_{n}^{\prime \prime l}\right)\left\|x_{n}^{l}\right\| \exp (j \omega t) ; \\
u_{-n}^{l} & =\left(\hat{u}_{n}^{\prime l}-j \hat{u}_{n}^{\prime \prime l}\right)\left\|x_{-n}^{l}\right\| \exp (-j \omega t),
\end{aligned}
$$

where $\hat{u}_{n}^{\prime l}$ and $\hat{u}_{n}^{\prime \prime l}$ are twin mode shapes associated to the eigenfrequencies $\omega_{n}^{l}$ in the stationary wave basis and are function of the cylindrical co-ordinates, with $l$ the index of the twin mode, and where \|\| denotes the modulus of the generalized co-ordinates $x_{n}^{l}$ and $x_{-n}^{l}$ in the rotating wave basis. Then the displacement vector in Eq. (3) can be written as

$$
u=\sum_{n} \sum_{l}\left(x_{n}^{\prime \prime} \hat{u}_{n}^{\prime l}+j x_{n}^{\prime \prime \prime} \hat{u}_{n}^{\prime \prime l}\right)
$$

In this Eq. (6), $x_{n}^{\prime l}$ and $j x_{n}^{\prime \prime l}$ are the generalized coordinates associated to the mode shapes $\hat{u}_{n}^{\prime l}$ and $\hat{u}_{n}^{\prime \prime l}$.

Let us consider firstly the structural response under an arbitrary excitation force $f(t)$ acting at a point $P$ and time $t$ on the cyclic symmetric fluidstructure boundary $\Gamma$. This excitation force $f(t)$ being developable in Fourier series (see Eq. (8)), then equilibrium equations for a non-dissipative system in stationary wave basis may be written as

$$
\begin{aligned}
\ddot{x}_{n}^{\prime i}+\left(\omega_{n}^{i}\right)^{2} x_{n}^{\prime i}=\int_{\Gamma} \hat{u}_{n}^{\prime i} \cdot f(t) \mathrm{d} s & \forall i=1, \ldots, \infty, \\
\ddot{x}_{n}^{\prime \prime i}+\left(\omega_{n}^{i}\right)^{2} x_{n}^{\prime \prime i}=\int_{\Gamma}-j \hat{u}_{n}^{\prime \prime i} \cdot f(t) \mathrm{d} s & \forall n=0, \ldots, \tilde{N},
\end{aligned}
$$

where the centred dot denotes the scalar product and the overdot denotes $\mathrm{d} / \mathrm{d} t$. In Eq. (7), $i$ is a positive integer, and from Eq. (3) $n=0, \ldots,+\tilde{N}$.

Here, the excitation force $f$ will be generated from the fluid flow for which no assumption is made, except the small disturbances. Denoting the external unitary normal on $\Gamma$ at the point $P$ by $m$, the pressure $p$ at the point $\mathrm{P}$ and time $t$ can be introduced and we have $f=p m$. Excitation forces in turbomachinery, generally come from flow distortions generated by blades and vanes and distortion of inlet. They can be observed in rotatory co-ordinate system with respect to the cyclic symmetric structure. 
Then, a Fourier series development of the excitation force $f$ may be introduced with respect to the circumferential co-ordinate $\theta-\bar{\omega} t$ associated to a rotatory co-ordinate system at the angular speed $\bar{\omega}$, and the flow distortion is

$$
f(t)=\sum_{q=-\infty}^{+\infty} f_{q}(r, z) \exp j q(\theta-\bar{\omega} t) .
$$

Using the basis changing between stationary and rotating wave bases, generalized co-ordinates with respect to the rotating wave basis can be expressed as follows:

$$
x_{n}^{i}=\frac{x_{n}^{\prime i}+x_{n}^{\prime \prime i}}{2}, \quad x_{-n}^{i}=\frac{x_{n}^{\prime i}-x_{n}^{\prime \prime i}}{2} .
$$

Thus from Eq. (7), equilibrium equations of the cyclic periodic structure in rotating wave basis for a non-dissipative system are deduced:

$$
\begin{aligned}
\ddot{x}_{n}^{i} & +\left(\omega_{n}^{i}\right)^{2} x_{n}^{i} \\
& =\frac{1}{2} \int_{\Gamma} \hat{u}_{n}^{i i} \cdot f(t) \mathrm{d} s-\frac{1}{2} \int_{\Gamma} j \hat{u}_{n}^{\prime \prime i} \cdot f(t) \mathrm{d} s, \\
\ddot{x}_{-n}^{i} & +\left(\omega_{n}^{i}\right)^{2} x_{-n}^{i} \\
& =\frac{1}{2} \int_{\Gamma} \hat{u}_{n}^{\prime i} \cdot f(t) \mathrm{d} s+\frac{1}{2} \int_{\Gamma} j \hat{u}_{n}^{\prime \prime i} \cdot f(t) \mathrm{d} s .
\end{aligned}
$$

It can be shown (Wildheim, 1979; Lalanne, 1989) that resonance condition requires

$$
\omega_{n}^{i}=q \bar{\omega}=(k N \pm n) \bar{\omega}
$$

for all positive integers $k, n$, taking into account the inequality $n \leq N / 2$. Since the system considered is non-dissipative, the resonance condition is reached when excitation frequency becomes equal to any eigenfrequency, and the generalized forces in Eq. (10) then being non-zero.

Let us suppose that the aeroelastic load is generated from the displacement $u$ at the boundary and that now this load satisfies cyclic symmetric conditions. In the rotating wave basis, we can write

$$
\begin{aligned}
u & =\sum_{n} \sum_{l}\left[x_{n}^{l}\left(\hat{u}_{n}^{\prime l}+j \hat{u}_{n}^{\prime \prime l}\right)+x_{-n}^{l}\left(\hat{u}_{n}^{\prime l}-j \hat{u}_{n}^{\prime \prime \prime}\right)\right] \\
& =\sum_{n} \sum_{l}\left(u_{n}^{l}+u_{-n}^{l}\right) .
\end{aligned}
$$

Within the linearity assumption kept here, a harmonic excitation at circular frequency $\omega$ involves identical frequency harmonic load. Thus, in the case wherein the general excitation load $f(t)$ is an aeroelastic one, we can write for a harmonic motion

$$
f_{n}(j \omega)=\sum_{l} \hat{f}_{n}^{l}(r, z, \theta, j \omega) x_{n}^{l}(j \omega),
$$

where

$$
\begin{aligned}
x_{n}^{l}(j \omega) & =\left\|x_{n}^{l}\right\| \exp (j \omega t), \\
x_{-n}^{l}(j \omega) & =\left\|x_{-n}^{l}\right\| \exp (-j \omega t) \quad \text { and } \\
\hat{f}_{n}^{l}(r, z, \theta, j \omega) & =-p_{n}^{l}(r, z, \theta, j \omega) m .
\end{aligned}
$$

The generalized force $f_{n}^{l i}(j \omega)$ generated by the harmonic displacement $u_{n}^{l}(j \omega)$ for the $i$ th wave of generalized co-ordinate $x_{n}^{i}(j \omega)$ is given by

$$
f_{n}^{l i}(j \omega)=h_{n}^{l i}(j \omega) \pi \rho b^{2} \omega^{2} x_{n}^{l}(j \omega),
$$

with

$$
\begin{aligned}
& \pi \rho b^{2} \omega^{2} h_{ \pm n}^{l i}(j \omega) \\
& \quad=\frac{1}{2} \int_{\Gamma}-p_{n}^{l}(r, z, \theta, j \omega) m \cdot\left[\hat{u}_{n}^{\prime i} \mp j \hat{u}_{n}^{\prime \prime i}\right] \mathrm{d} s .
\end{aligned}
$$

In these last expressions, $\rho$ is the mass density of the fluid, $b$ the mid-chord or a characteristic size, and then $b \omega / V$ is the Strouhal number while $V$ is velocity far away in the fluid flow. $h_{n}^{l i}(j \omega)$ is the complex amplification factor at the frequency $\omega$.

The generalized loads in Eq. (10) can now be explicited using a time harmonic analysis based on decomposition associated with the generalized coordinates $x_{n}^{l}$ and their successive derivatives 
$\dot{x}_{n}^{l}, \ddot{x}_{n}^{l}, \dddot{x}_{n}^{l}, \ldots$ Some additional state variables may be added to include the delay effects and acoustic resonances (see Poiron, 1995; Crawley, 1988). From an identification procedure between definition of the aerodynamic loading (see Eq. (7) for example) and the above introduced time harmonic analysis, the generalized forces in Eq. (10) can be written as

$$
\begin{aligned}
& \int_{\Gamma} \hat{u}_{n}^{\prime i} \cdot f(t) \mathrm{d} s \\
& =\sum_{l}\left(a_{n} x_{n}^{\prime l}+b_{n} \dot{x}_{n}^{\prime l}+c_{n} \ddot{x}_{n}^{\prime l}+\cdots\right. \\
& \left.\quad+\tilde{a}_{n} x_{n}^{\prime \prime l}+\tilde{b}_{n} \dot{x}_{n}^{\prime \prime l}+\tilde{c}_{n} \ddot{x}_{n}^{\prime \prime l}+\cdots\right), \\
& -\int_{\Gamma} j \hat{u}_{n}^{\prime \prime \prime} \cdot f(t) \mathrm{d} s \\
& =\sum_{l}\left(a_{n} x_{n}^{\prime \prime l}+b_{n} \dot{x}_{n}^{\prime \prime l}+c_{n} \ddot{x}_{n}^{\prime \prime l}+\cdots\right. \\
& \left.\quad+\tilde{a}_{n} x_{n}^{\prime l}+\tilde{b}_{n} \dot{x}_{n}^{\prime l}+\tilde{c}_{n} \ddot{x}_{n}^{\prime l}+\cdots\right),
\end{aligned}
$$

$a_{n}, \tilde{a}_{n}, b_{n}, \tilde{b}_{n}, c_{n}, \tilde{c}_{n}, \ldots$, being complex numbers and where indexes $i, l$ have been omitted. These coefficients are depending on the aeroelastic properties of the fluid structure given by Eqs. (13)-(16). In Eq. (10), aeroelastic quantities modify the dynamics of the system, but from the orthogonality properties, Eq. (10) associated to the phase difference index $n$ are formally unchanged and can be separately solved. This is not true in the modal basis.

Using Eq. (4), the aeroelastic problem (10)-(17) could be defined on the reference sector $\Omega_{\mathrm{o}}$ and in the rotating wave basis.

\section{SPECIAL CASES}

\section{Weak Aeroelastic Coupling}

The fluid is light, and then it is assumed that the aeroelastic forces are small by comparison with that of mechanical ones. Aeroelastic loads are only significant near resonances, then, they will be given by

$$
a_{n} x_{n}^{\prime l}+b_{n} \dot{x}_{n}^{\prime l}+\tilde{a}_{n} x_{n}^{\prime \prime l}+\tilde{b}_{n} \dot{x}_{n}^{\prime \prime l}
$$

and

$$
a_{n} x_{n}^{\prime \prime l}+b_{n} \dot{x}_{n}^{\prime \prime l}+\tilde{a}_{n} x_{n}^{\prime l}+\tilde{b}_{n} \dot{x}_{n}^{\prime l}
$$

associated to in-phase and quadrature terms in the harmonic motion. Indexes $i, l$ are omitted before, and coefficients $a_{n}, b_{n}, \tilde{a}_{n}, \tilde{b}_{n}$ are now real numbers.

\section{Rigid Disk}

In this case, $u_{n}^{i}=u_{p}^{i}$ for all $n$ and $p$, and for all point in the reference sector $\Omega_{\mathrm{o}}$. From Eq. (4)

$$
u^{i}\left(r, \theta_{0}+\frac{2 \pi s}{N}, z, t\right)=u^{i}\left(r, \theta_{0}, z, t\right) \exp (2 \pi j n s / N) \text {. }
$$

The linearity assumption, in addition to the rigid disk one, allows to obtain pressure on the reference sector during motion as the superimposition of pressures on each other sector. Thus, the harmonic motion involves

$$
\begin{gathered}
\hat{f}_{n}^{i}(r, z, \theta)=\sum_{l}^{N} \hat{f}_{s}^{i} \exp (2 \pi j n s / N) \text { on } \Omega_{0} \\
\text { i.e. } \theta \in\left[\theta_{0}, \theta_{0}+\frac{2 \pi}{N}[.\right.
\end{gathered}
$$

\section{Blade Interaction}

From experiments and calculations, it is admitted that any blade is sensitive to the interactions produced from the motion of two adjacent blades on either side of the blade considered (Szechenyi, 1987). So, aeroelastic loading knowledge for five values of the phase difference index $n$ allows to compute load for any phase difference index.

\section{Efficient Strategy}

The above particular cases furnish a way to reduce computational cost from the Fourier analysis using the rotating basis. From the Eq. (4), where $u_{n}^{l}=u_{p}^{l}$ on $\Omega_{0}$, for all $n$ and $p$, the blade interaction assumption allows to keep only five values for the phase difference index. Then, mode decomposition should use the rotating wave basis. Finally, if the aeroelastic coupling is weak, stability analysis will be made near each resonance. 


\section{TWIN MODE APPROACH - WEAK AEROELASTIC COUPLING - STABILITY}

If roots of the twin modes are far, those latter will be almost uncoupled, when mode veering does not occur (Morand and Ohayon, 1992). From Eq. (19) and the above weak aeroelastic coupling assumption, the following equilibrium equations are found, in the rotating basis:

$$
\begin{aligned}
& \ddot{x}_{n}^{i}+\left(\alpha_{c n}^{i}+\beta_{c n}^{i}\right) \dot{x}_{n}^{i}+\left(\alpha_{k n}^{i}+\beta_{k n}^{i}\right) x_{n}^{i}=0, \\
& \ddot{x}_{-n}^{i}+\left(\alpha_{-c n}^{i}-\beta_{-c n}^{i}\right) \dot{x}_{-n}^{i}+\left(\alpha_{-k n}^{i}-\beta_{-k n}^{i}\right) x_{-n}^{i}=0,
\end{aligned}
$$

with

$$
\begin{aligned}
& \alpha_{c n}^{i}=2 \omega_{n}^{i} \xi_{n}^{i}+\sum_{s=0}^{N-1} 2 \alpha_{n}^{s i} \cos n s \alpha, \\
& \alpha_{k n}^{i}=\left(\omega_{n}^{i}\right)^{2}+\sum_{s=0}^{N-1} \gamma_{n}^{s i} \cos n s \alpha, \\
& \beta_{c n}^{i}=\frac{1}{\omega_{n}^{i}} \sum_{s=0}^{N-1} \gamma_{n}^{s i} \sin n s \alpha, \\
& \beta_{k n}^{i}=2 \omega_{n}^{i} \sum_{s=0}^{N-1} \alpha_{n}^{s i} \sin n s \alpha .
\end{aligned}
$$

According to the above weak aeroelastic coupling assumption, we have the relations

$$
\begin{aligned}
& -a_{n}^{i}=\alpha_{k n}^{i}-\left(\omega_{n}^{i}\right)^{2}, \quad-b_{n}^{i}=\alpha_{c n}^{i}-2 \omega_{n}^{i} \xi_{n}^{i}, \\
& -\tilde{a}_{n}^{i}=\beta_{k n}^{i}, \quad-\tilde{b}_{n}^{i}=\beta_{c n}^{i} .
\end{aligned}
$$

In Eq. (21), coefficients have the following physical sense:

- $\alpha_{c n}^{i}, \beta_{c n}^{i}$ are viscous and pseudo-viscous aeroelastic terms,

- $\alpha_{n}^{s i} \quad$ are in-phase force terms due to motion of the sector $\Omega_{s}$ at the mode $i$,

- $\xi_{n}^{i} \quad$ are viscous damping coefficients,

- $\gamma_{n}^{s i} \quad$ are in quadrature force terms generated from the motion of the sector $\Omega_{s}$ at the mode $i$,

- $\alpha_{k n}^{i} \beta_{k n}^{i}$ are stiffness and pseudo-stiffness aeroelastic terms.

Example As an example, we consider now a bladed-disk incorporating a rigid disk surrounded by 20 blades allowing the cyclic symmetry assumption available. Blade vibrations may be bending, torsion or any other mode type. Hereafter are given data corresponding to an abstract problem useful only to analyse trends for the dynamic behaviour of the fluid-structure system.

Aeroelastic interaction concerns only one sector adjacent to the reference sector. Thus, required terms are restricted in Eq. (21) to $s$ values $N-1,0,1$. Non-zero coefficients are given by

$$
\begin{array}{lll}
\alpha^{0}=5.72 \mathrm{~s}^{-1}, & \alpha^{1}=10 \mathrm{~s}^{-1}, & \alpha^{N-1}=2 \mathrm{~s}^{-1}, \\
\gamma^{0}=0.01 \mathrm{~s}^{-2}, & \gamma^{1}=75000 \mathrm{~s}^{-2}, & \gamma^{1}=25000 \mathrm{~s}^{-2}, \\
\omega_{n}^{i}=6283 \mathrm{rad} \mathrm{s}^{-1}, & N=20, & \zeta=0.001,
\end{array}
$$

where the indexes $n$ and $i$ are omitted.

Root calculation from the characteristic equation of the differential system (20), having exponential solutions, allows to determine stable and unstable zone in the complex plane, see Fig. 1. Modes corresponding to phase difference indexes $n=8$ and 9 are unstable. The Routh-Hurwitz criterion also gives the same following stability condition:

$$
\left(\alpha_{c n}^{i}\right)^{2}-\left(\beta_{c n}^{i}\right)^{2}>0 \text { and } \alpha_{c n}^{i}>0
$$

\section{SMALL MISTUNING BETWEEN SECTORS FOR AN AEROELASTIC PROBLEM}

The twin mode approach is still supposed to be available in modal basis. So, corresponding equilibrium equations for an aeroelastic problem are deduced from Eq. (20). By adding the basis change defined in Eq. (9), they are finally given in the stationary wave basis as

$$
\begin{aligned}
& \ddot{x}_{n}^{\prime i}+\alpha_{c n}^{i} \dot{x}_{n}^{\prime i}+\beta_{c n}^{i} \dot{x}_{n}^{\prime \prime i}+\alpha_{k n}^{i} x_{n}^{\prime i}+\beta_{k n}^{i} x_{n}^{\prime \prime i}=0, \\
& \ddot{x}_{n}^{\prime \prime i}+\alpha_{c n}^{i} \dot{x}_{n}^{\prime \prime i}+\beta_{c n}^{i} \dot{x}_{n}^{\prime i}+\alpha_{k n}^{i} x_{n}^{\prime \prime i}+\beta_{k n}^{i} x_{n}^{\prime i}=0 .
\end{aligned}
$$

Assuming a small mistuning between sectors of a cyclic periodic structure, it is still possible to restrict stability analysis to twin mode formulation as previously suggested in Wei and Pierre (1988). Here 


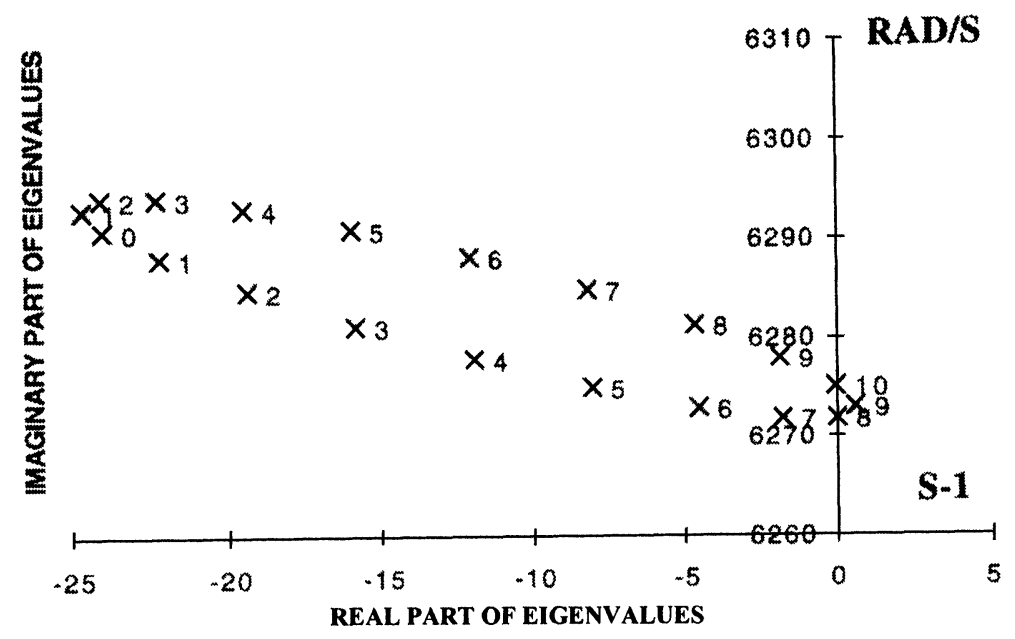

FIGURE 1 Stability of the tuned system.

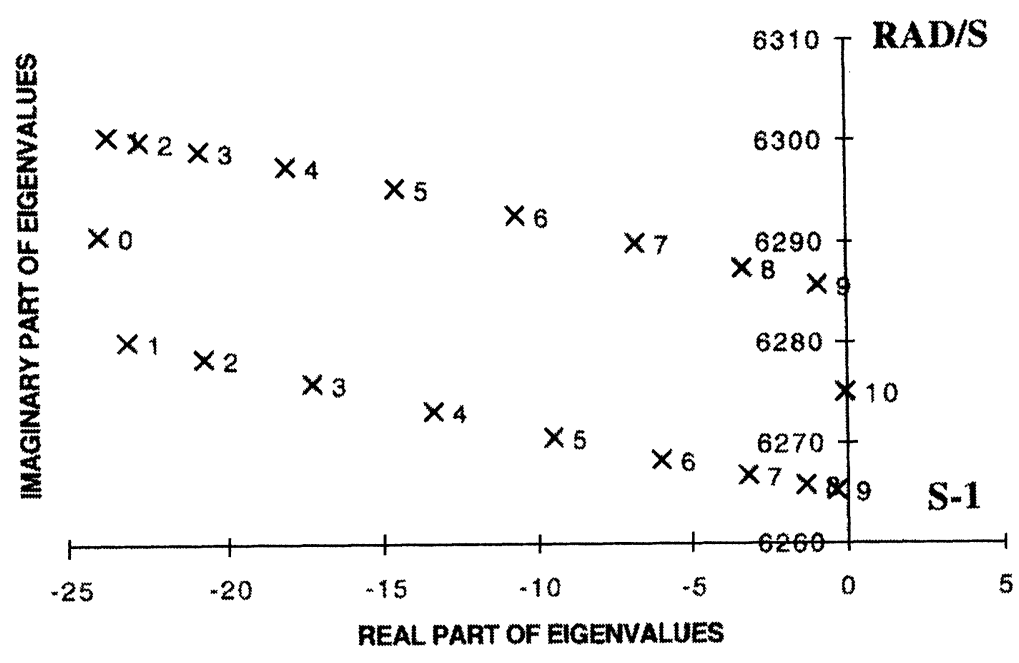

FIGURE 2 Stability of the mistuned system.

coupling comes from aerodynamics. Thus, considering a small perturbation $\Delta h_{n}^{i}$ of the coefficient $\alpha_{k n}^{i}$, the coefficient $\left(\alpha_{k n}^{i}+\Delta h_{n}^{i}\right)$ is substituted to $\alpha_{k n}^{i}$ in one of the Eq. (23). Then, the corresponding eigenvalue equation is usually written. Finally, the Routh-Hurwitz criterion is used and the stability condition is derived as follows:

$$
\begin{aligned}
& \left(\alpha_{c} \Delta h\right)^{2}+2\left[\alpha_{c}^{2}-\beta_{c}^{2}\right]\left[2 \beta_{k}^{2}-2 \alpha_{c} \beta_{k}\right. \\
& \left.\quad+\alpha_{c}^{2}\left(2 \alpha_{k}+\Delta h\right)\right]>0 \text { and } \alpha_{c}>0
\end{aligned}
$$

where indexes $i$ and $n$ are omitted.
It has been proved that stability margin increases when the mistuning occurs. Next, a mistuning to the above example showed in previous section is applied as follows:

$$
\omega_{n}^{i}=6273 \mathrm{rad} \mathrm{s}^{-1} \text { and } \omega_{n}^{i}+\frac{\Delta h_{n}^{i}}{2}=6293 \mathrm{rad} \mathrm{s}^{-1} .
$$

Then, the system becomes stable as shown in Fig. 2.

Physically, if we introduce some small lack of symmetry into the assembly, the twin modes no longer have the same frequency. It follows that the 
unsteady forces caused by either of the modes no longer have the ideal frequency for exciting the other.

\section{CONCLUDING REMARKS}

Using the twin mode approach, the linearized approximation given for aeroelastic problems encountered in turbomachinery, having light fluid in which turbine is immersed, has allowed to recover resonance conditions and to derive stability conditions, when cyclic symmetric properties are satisfied.

From general conditions associated to cyclic symmetric structures with aeroelastic loading based on weak aeroelastic coupling, $N$ independant stability problems defined in the reference sector $\Omega_{0}$ are deduced in rotating wave basis, according to the $N$ values of the phase difference index associated to the $N$ sectors of the structure considered.

A stability study is proposed when a small mistuning occurred between sectors of an aeroelastic cyclic symmetric structure. The solution is based on a perturbation technique. It has been established that an unstable, perfectly, periodic structure can become stable with a small mistuning between sectors of this structure.

\section{References}

Balasubramanian, P., Jagadeesh, J.G., Suhas, H.K., Srivastava, A.K. and Ramamurti, V. (1993) On the use of the Ritz-Wilson method for the dynamic response analysis of cyclic symmetric structures, Journal of Sound and Vibration, 164(2), 193-206.
Childs, D. (1993) Turbomachinery Rotordynamics, John Wiley and Sons, New York, Chichester, Brisbane, Toronto, Singapore.

Crawley, E.F. (1988) Aeroelastic formulation for tuned and mistuned rotors, AGARD A-G 298, Vol. 2.

Ewins, D.J. (1988) Structural dynamic characteristics of bladed assemblies, AGARD, A-G 298, Vol. 2

Ince, E.L. (1956) Ordinary Differential Equations, Dover Publications, Inc., New York.

Jacquet-Richardet, G. and Henry, R.A. (1994) Modal aeroelastic finite element analysis method for advanced turbomachinery stages, International Journal for Numerical Methods in Engineering, 37, 4205-4217.

Lalanne, B. (1989) Vibrations of cyclic symmetric structures. Modes - Resonances - Damping - Stability - Control (in French), 9th French Congress of Mechanics, Metz.

Lalanne, B. and Touratier, M. (1996) Friction damping of blade vibrations of gas turbines (in French), DRET Report of the Grant, No. 92/471.

Lane, F. (1956) System mode shapes in the flutter of compressor blade rows, Journal of the Aeronautical Sciences, 23, 65-66.

Marshall, J.G. and Imregun, M. (1996) Review of aeroelasticity methods with emphasis on turbomachinery applications, Journal of Fluid and Structures, 10(3), 237-267.

Mézière, L. (1994) Vibrations of cyclic symmetric structures Application to turbomachines (in French), AGARD, CP 537 Vol. 1.

Morand, H.J.P. and Ohayon, R. (1992) Fluid Structure Interactions (in French), Masson, Paris, Milan, Barcelone, Bonn

Poiron, F. (1995) Time model in aeroservoelasticity systems. Application to time delay effects (in French), La Recherche Aérospatiale, No. 2, 103-114.

Stocker, J.J. (1992) Non-Linear Vibrations in Mechanical and Electrical Systems, John Wiley and Sons, Inc., New York.

Szechenyi, E. (1987) Understanding fan blade flutter through linear cascade aeroelastic testing, AGARD A-G 298, Vol. 1

Thomas, D.L. (1974) Standing waves in rotationally periodic structures, Journal of Sound and Vibration, 37, 288-290.

Touratier, M. (1986) Floquet waves in a body with a slender periodic structure, Wave Motion, 8, 485-495.

Wagner, L.F. and Griffin, J.H. (1994) Forced harmonic response of grouped blade system: Part I - discrete theory, International Gas Turbine and Aeroengine Congress and Exposition, ASME, 94-GT-203, The Hague.

Wei, S.T. and Pierre, C. (1988) Localization phenomena in mistuned assemblies with cyclic symmetry, Trans. ASME, Journal of Vibration, Acoustics, Stress and Reliability in Design, 110(4), 61-79.

Wildheim, S.J. (1979) Excitations of rotationally periodic structures, Trans. ASME, Journal of Applied Mechanics, 46, 878-882. 


\section{ait \\ ENERGY MATERIALS}

M A N E Y publishing

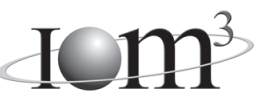

\section{Materials Science \& Engineering for Energy Systems}

Maney Publishing on behalf of the Institute of Materials, Minerals and Mining

The Institute of Materials, Minerals \& Mining

Economic and environmental factors are creating ever greater pressures for the efficient generation, transmission and use of energy. Materials developments are crucial to progress in all these areas: to innovation in design; to extending lifetime and maintenance intervals; and to successful operation in more demanding environments. Drawing together the broad community with interests in these areas, Energy Materials addresses materials needs in future energy generation, transmission, utilisation, conservation and storage. The journal covers thermal generation and gas turbines; renewable power (wind, wave, tidal, hydro, solar and geothermal); fuel cells (low and high temperature); materials issues relevant to biomass and biotechnology; nuclear power generation (fission and fusion); hydrogen generation and storage in the context of the 'hydrogen economy'; and the transmission and storage of the energy produced.

As well as publishing high-quality peer-reviewed research, Energy Materials promotes discussion of issues common to all sectors, through commissioned reviews and commentaries. The journal includes coverage of energy economics and policy, and broader social issues, since the political and legislative context influence research and investment decisions.

\section{CALL FOR PAPERS}

Contributions to the journal should be submitted online at http://ema.edmgr.com

To view the Notes for Contributors please visit: www.maney.co.uk/journals/notes/ema

Upon publication in 2006, this journal will be available via the Ingenta Connect journals service. To view free sample content online visit: www.ingentaconnect.com/content/maney

For further information please contact:

Maney Publishing UK

Tel: +44 (0)113 2497481 Fax: +44 (0)1132486983 Email: subscriptions@maney.co.uk

or

Maney Publishing North America

Tel (toll free): 8662975154 Fax: 6173546875 Email: maney@maneyusa.com

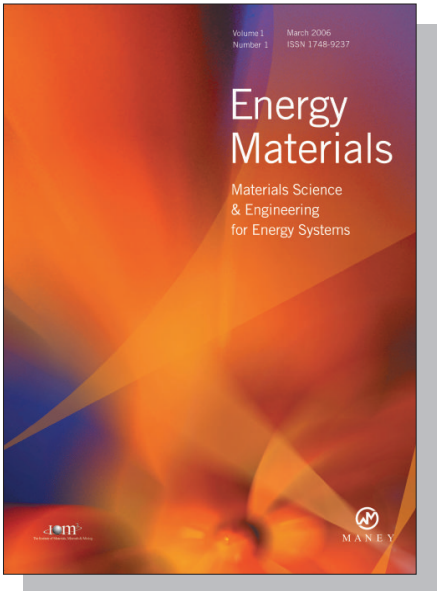

EDITORS

Dr Fujio Abe

NIMS, Japan

Dr John Hald, IPL-MPT, Technical University of Denmark, Denmark

Dr R Viswanathan, EPRI, USA

\section{SUBSCRIPTION INFORMATION}

Volume 1 (2006), 4 issues per year

Print ISSN: 1748-9237 Online ISSN: 1748-9245

Individual rate: $£ 76.00 / U S \$ 141.00$

Institutional rate: $£ 235.00 /$ US $\$ 435.00$

Online-only institutional rate: $£ 199.00 / U S \$ 367.00$

For special $\mathrm{IOM}^{3}$ member rates please email

subscriptions@maney.co.uk

\section{For further information or to subscribe online please visit www.maney.co.uk}



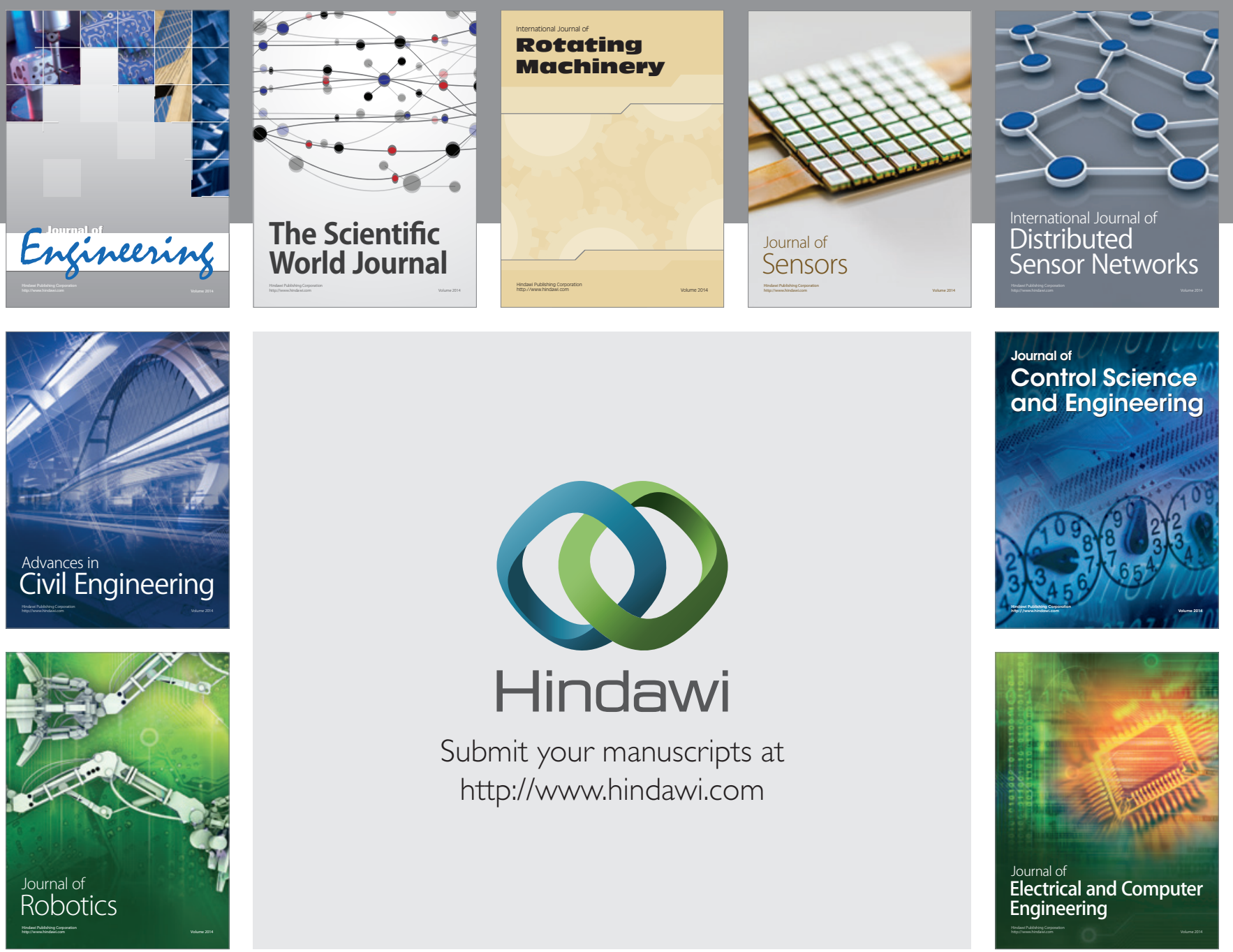

Submit your manuscripts at

http://www.hindawi.com
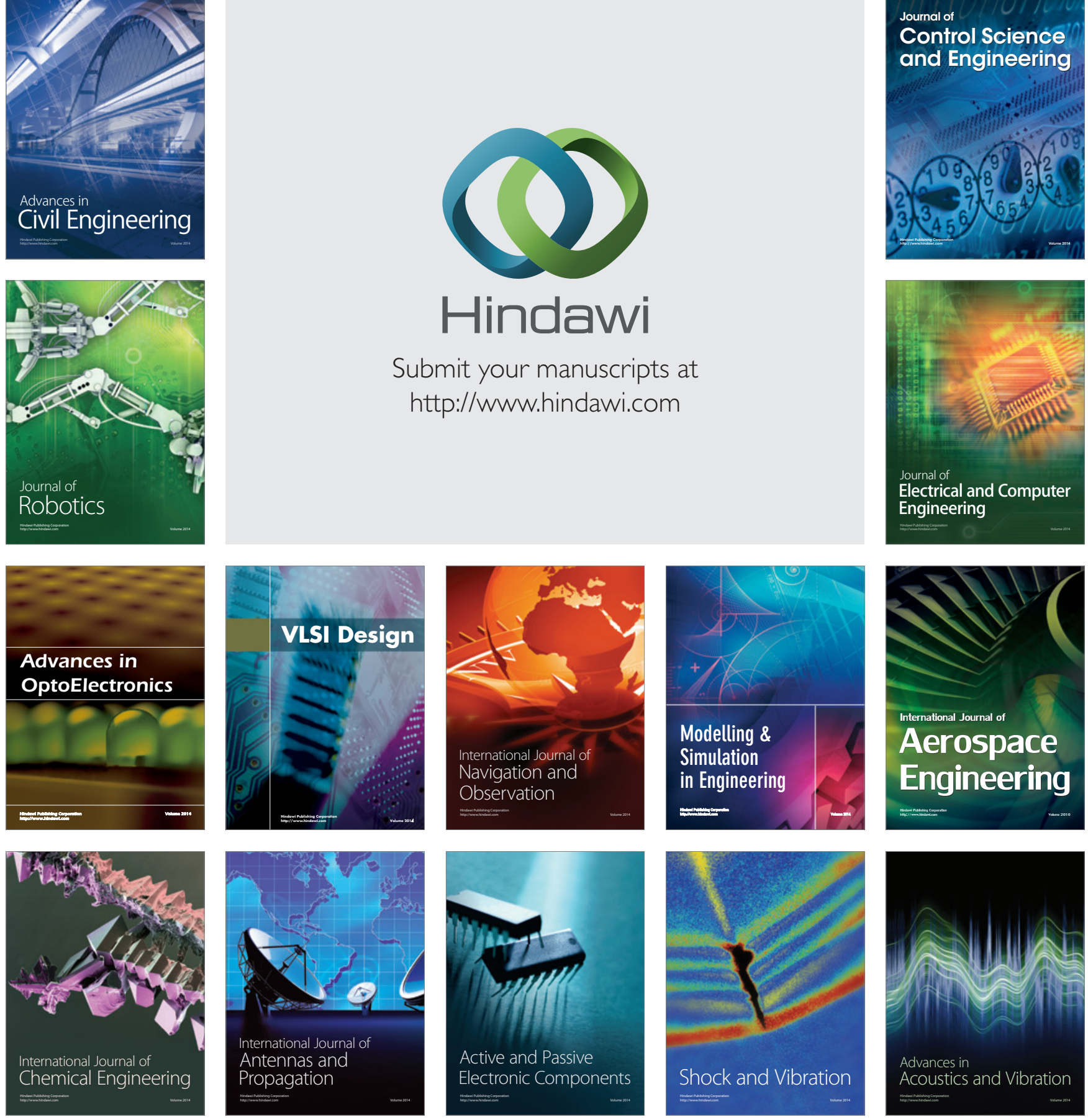